\title{
HIGHER ORDER ARCHITECTURE OF COLLECTIONS OF OBJECTS
}

\author{
NILS A. BAAS* \\ Deparment of Mathematical Sciences, NTNU, N-7491 Trondheim, Norway
}

\begin{abstract}
We show that on an arbitrary collection of objects there is a wide variety of higher order architectures governed by hyperstructures. Higher order gluing, local to global processes, fusion of collections, bridges and higher order types are discussed. We think that these types of architectures may have interesting applications in many areas of science.
\end{abstract}

Keywords: Hyperstructure, higher order architecture, collection, hypercategory, sheaf, globalizer, bonds and bridges, types.

\section{INTRODUCTION}

In all human activites we consider objects and collections of objects. We study how we can make new objects and collections out of old ones by using their properties, relations and interactions. In order to do so it is important to have in mind what kind of architectures to use when forming new structures and organizations out of given collections of objects. What are the possibilities?

The main purpose of this paper is to describe new architectures and discuss how to organize a collection of objects in theory and practice. We will introduce higher order architectures which lead to new and unexplored structures and organizations extending for example categorical organizations. We basically follow the hyperstructure idea with a new extension. We will discuss examples and situations where these new architectures may be useful.

Our main purpose is to point out that there is a plethora of higher order architectures waiting to be explored.

\section{Architectures}

When we are given a collection of objects and we want to organize them, what are the architectures we may use?

Let us think of an arbitrary collection $\mathscr{C}$ as a set of objects. In settings where we would like to avoid set theory the general idea will apply as well.

\footnotetext{
*Email: baas@math.ntnu.no.
} 
Finite collections can be organized in many ways. Given a finite set $X$ we may consider the elements or objects as vertices in a graph or a network if we assign weights on the edges. We may extend this to hypergraphs where the edges are replaced by subsets of objects instead of pairs.

Another useful organization of a set $X$ is as a simplicial complex which is specified by a set of finite non-empty subsets $S \subset \mathcal{P}(X)$ (the power set of $X$, i.e. the set of all subsets) such that if $s \in S$ and $t \subset s$ ( $t$ non-empty), then $t \in S$ and $x \in X$ implies $\{x\} \in S$.

A topological space is an organization of a set $X$ which is given by a subcollection $\mathcal{T} \subset \mathcal{P}(X)$ satisfying certain axioms. The elements of $\mathcal{T}$ are called open sets.

Similarly a measure space is defined by another type of subcollection $\mathcal{A} \subset \mathcal{P}(X)$ satisfying a different set of axioms. Many types of structures can be described in this way as in Bourbaki (1966).

A category is an organization on a collection of objects (class or set) where one assigns a set

$$
\operatorname{Mor}(X, Y)
$$

to every ordered pair of objects. In addition there is a composition law.

We will define an organizational architecture which encompasses these examples.

The basic idea: Instead of assigning a set $\operatorname{Mor}(X, Y)$ to every ordered pair of objects we will assign a set of bonds to any collection of objects - finite, infinite or uncountable:

$$
\operatorname{Bond}(X, Y, Z, \ldots) \text { or } \operatorname{Bond}(c \in \mathscr{C})
$$

$\mathscr{C}$ being a collection or parametrized family of objects. We may also consider ordered collections or collections with other additional properties. Bonds extend morphisms in categories and higher bonds create levels and extend higher morphisms (natural transformations and homotopies, etc.). This will be the basis for the creation of new global states.

More formally our extended architecture is as follows:

$X$ is a set representing an arbitrary collection of objects. Then we consider the iterated power sets

$$
X, \mathcal{P}(X), \mathcal{P}^{2}(X), \ldots, \mathcal{P}^{k}(X), \ldots
$$

For $S \in \mathcal{P}^{k}(X)$ for some $k$ we assign a set of properties or states as follows:

$$
\Omega: \mathcal{P}^{k}(X) \rightarrow \text { Sets }
$$

and we think of $\Omega$ as a presheaf functor in a suitable way. Let us put

$$
\mathscr{P}(X)=\bigcup_{k=0}^{\infty} \mathcal{P}^{k}(X)
$$


and extend

$$
\Omega: \mathscr{P}(X) \rightarrow \text { Sets }
$$

The philosophy is that we first pick out the generalized subset we want to bind, then we assign the properties that will be involved in the binding process ending up with pairs

$$
(S, \omega) \quad S \in \mathscr{P}(X) \text { and } \omega \in \Omega(S) .
$$

In general we may also consider situations where $\mathscr{P}(X)$ is a suitable space of collections of spaces, algebras, etc. This means that $\mathscr{P}(X)$ may be a space where for example the points are spaces as well, like in moduli type spaces.

A typical $S$ may look like the configuration in Figure 1 .

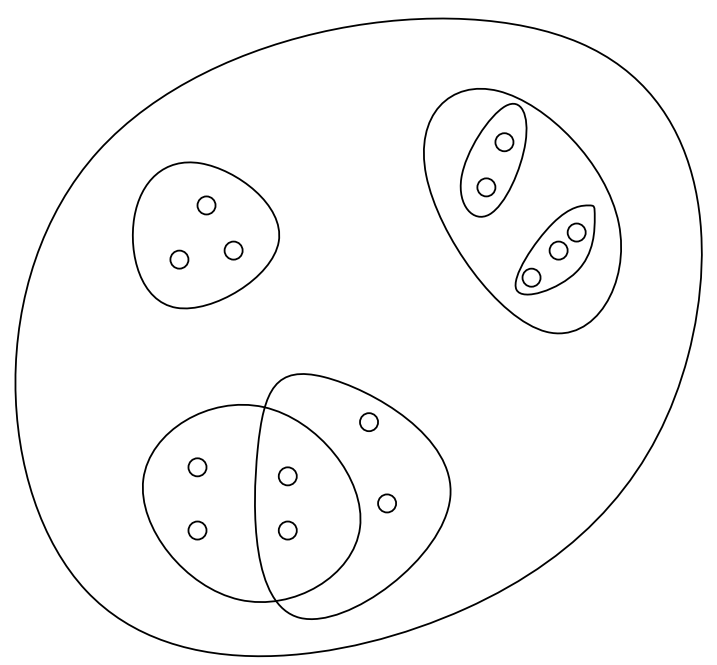

Figure 1: A typical generalized collection

These pairs are now our building blocks for new collections and we have to specify how we bind them together. Formally as for the original hyperstructures we define

$$
\Gamma=\{(S, \omega) \mid S \in \mathscr{P}(X) \text { and } \omega \in \Omega(S)\}
$$

$B$ is then an assignment of a set of bonds

$$
B: \Gamma \rightarrow \text { Sets }
$$

which we also may think of as a presheaf functor in a suitable way. Clearly some binding sets may be empty if the collection does not bind under the present circumstances.

This extends notions of morphisms, relations, etc. 


\section{Higher order ARCHitectures}

What about using the bonds of collections as building blocks for new bonds of collections of collections?

This is a natural extension in order to create higher order structures similar to higher power sets $-\mathcal{P}^{k}(X)$, relations of relations, morphisms of morphisms in higher category theory.

Let us consider an example. Suppose that we are given a finite set of objects $V$. In a given context we want to maximize the number of interactions in $V$. The optimal structure is clearly the complete graph, which may not always be obtainable, so in general we end up with a subgraph of the complete graph. Does the evolutionary process of creating more and more interactions stop here?

No, because we may let subsets of $V$ - not just pairs - interact. When we have exhausted this possibility, we may proceed to subsets of subsets, etc. This shows that even on a finite set there is an enourmous number of possibilities of evolving new interactions. We use this example as a guide for the general contruction.

In describing higher order architectures of organizations of collections we follow the "General Principle" of hyperstructures in Baas (2006), for background material see (Baas 1994a, Baas 1994b). The objects may be abstract or physical.

This means that we start with a basic collection $\mathscr{C}$. As in (Baas 2006, Baas 2009b, Baas 2013b) we set $\mathscr{C}=X_{0}$, and then form successively:

$$
X_{0}, \quad \Omega_{0}, \quad \Gamma_{0}, \quad B_{0} .
$$

In order to create the next level we put

$$
X_{1}=\left\{b_{0} \mid b_{0} \in B_{0}\left(S_{0}, \omega_{0}\right), S_{0} \in \mathscr{P}\left(X_{0}\right) \text { and } \omega_{0} \in \Omega_{0}\left(S_{0}\right)\right\} .
$$

Depending on the situation we now can choose $\Omega_{1}$ and $B_{1}$ according to what we want to construct or study and then repeat the construction.

This is not a recursive procedure since new properties and bonds arise at each level.

Hence a higher order architecture of order $n$ is described by:

$$
\mathscr{H}_{n}:\left\{\begin{array}{cccc}
X_{0}, & \Omega_{0}, & \Gamma_{0}, & B_{0} \\
X_{1}, & \Omega_{1}, & \Gamma_{1}, & B_{1} \\
& \vdots \\
X_{n}, & \Omega_{n}, & \Gamma_{n}, & B_{n} .
\end{array}\right.
$$

At the technical level we require that

$$
B_{i}\left(S_{i}, \omega_{i}\right) \cap B_{i}\left(S_{i}^{\prime}, \omega_{i}^{\prime}\right)=\emptyset
$$

for $S_{i} \neq S_{i}^{\prime}$ ("a bond knows what it binds") in order to define the $\partial_{i}$ 's below, or we could just require that the $\partial_{i}$ 's exist. 
The level architectures are connected by "boundary" maps as follows:

$$
\partial_{i}: X_{i+1} \rightarrow \mathscr{P}\left(X_{i}\right)
$$

defined by

$$
\partial_{i}\left(b_{i+1}\right)=S_{i}
$$

and maps

$$
I_{i}: X_{i} \rightarrow X_{i+1}
$$

such that $\partial_{i} \circ I_{i}=\mathrm{id}$. $I_{i}$ gives a kind of "identity bond".

The extensions allowing bindings of subsets or subcollections of higher power sets add many new types of architectures of hyperstructures. See Baas (2009a) for examples.

The structures $\mathscr{H}_{n}$ is an extension of what we called hyperstructures in (Baas 2006, Baas 2009b, Baas 2013b) but we cover them by the same name. In order to understand higher order structures we introduce, describe, define and detect hyperstructures.

By selective choices of observational mechanisms of properties and states the combinatorics may be kept at a reasonable level.

The general higher order architectural pattern is:

Start with a collection $\mathscr{C}=\mathscr{C}_{0}$,

$$
\begin{aligned}
\mathscr{C}_{0} & =\operatorname{Coll}_{0} \\
\mathscr{C}_{1} & =\operatorname{Bond}_{0}\left(\operatorname{Coll}_{0}\right) \\
& \vdots \\
\mathscr{C}_{n} & =\operatorname{Bond}_{n-1}\left(\operatorname{Coll}_{n-1}\left(\ldots\left(\operatorname{Coll}_{0}\right) \ldots\right)\right)
\end{aligned}
$$

where $\operatorname{Coll}_{k} \subset \mathscr{C}_{k}$ has been selected.

Definition: A higher order architecture of order $n$ on a collection of objects $\mathscr{C}$ is given by an $\mathscr{H}_{n}$-structure on $\mathscr{C}$.

Furthermore, in the realization of such structures in physics, chemistry, biology, etc. there is "a lot of room" in the nano-dimensions as pointed out by R. Feynmann.

The conclusion to be made is that there are universes of new higher order architectures of collections of objects waiting to be explored both in mathematics and other sciences.

\section{ExAmples}

\section{Links}

A link is a disjoint union of embedded circles (or rings) in three dimensional space. They may be linked in many ways. We consider linking as a kind of geometrical or topological binding, see Figure 2 . 


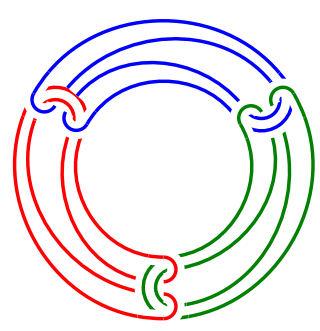

(a) Brunnian rings

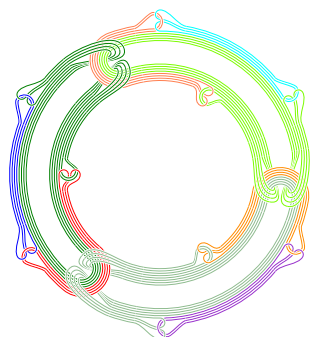

(b) 2nd order Brunnian rings

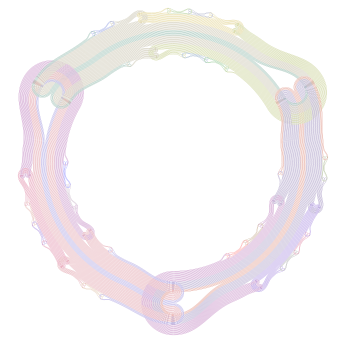

(c) 3rd order Brunnian rings

FigURE 2: Links

Once a link has been constructed we may inspect its connectivity properties and use these to form links of links - second order links.

We can then follow the general scheme that we have outlined for higher order architecture. Some of these have been extensively studied in Baas (2013a), especially with respect to Brunnian type binding properties.

\section{Molecules and materials}

The higher order architectures for geometric links may also act as architectures for molecules and how they form materials. In synthetic chemistry a lot of work has been done on the synthesis of Borromean rings. N. Seeman was the first to succeed using DNA molecules. In his laboratory work has now been started on the synthesis of second order Brunnian rings. The architectures we have outlined may be an interesting and useful guide for future synthesis. A challenging question is what kind of properties such materials will have and how they depend on the architecture. For a further discussion of these issues, see Baas (2009b), and Baas and Seeman (2012).

\section{Quantum states}

Many-body systems play an important role in physics, for example in quantum mechanics. For many-particle systems one may introduce the higher order architectures that we have discussed. However, can such an architecture be realized as a (bound) quantum state?

In 1970, the Russian physicist V. Efimov predicted new counter-intuitive quantum states where three particles are 
bound, but not two by two. This is analogous to the Borromean and Brunnian linking property. Experimentally such states were not observed until 2006 in ultracold caesium gases.

This raises the following interesting questions: Does there exist a family of quantum states analogous to higher order Brunnian links?

May all higher order architectures - for example for links - be realized as entangled quantum states? For a further discussion, see Baas (2013a) and Baas et al. (2014).

\section{Geometric bonds}

The idea is to extend higher cobordisms (cobordisms of cobordisms ...) and nested families of spaces to a hyperstructure context. We refer to Baas (2013b) for a discussion of this topic.

\section{Higher CATEgories AND HyPERCATEgories}

In category theory one organizes a collection of objects by assigning sets of morphisms to ordered pairs. In our terminology morphisms play the role of bonds - binding two objects together. Then for good mathematical reasons one introduces morphisms of morphisms - twomorphisms, up to general $n$-morphisms, etc. This is clearly a special case of the bonds of bonds ... situation.

However, it has turned out to be difficult to find a generally accepted definition of an $n$-category. From our point of view there is a recent approach (unpublished) by D. Ayala where higher categories are basically considered as sheaves on manifolds

$$
\operatorname{Sh}(M f d)
$$

In this approach the combinatorics of morphisms takes place on manifolds and the morphisms as bonds may geometrically be thought of as the cone on two points (source and target).

In our approach we want bonds to bind more than two objects: three, four,... even whole manifolds.

The cone in general introduces singularities (Figure 3).

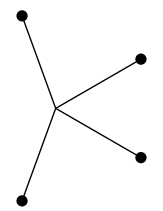

FiguRE 3: $\mathcal{C}\left(\mathbb{Z}_{4}\right)=$ cone on four points 
Intuitively it is a good and natural representation of a bond, and in general a cone on $P$ is a good example of a bond:

$$
\mathcal{C}(P)=\operatorname{Bond}\{p \mid p \in P\} .
$$

Let us therefore allow cone-type singularities. These have been studied in cobordism theory, Baas (1973), and we may even just consider arbitrary stratified sets. They have singular points, but can be decomposed in a regular way into smooth non-singular pieces called strata.

Locally any singular point look like the cone on a cone on a cone ... This geometrically iterated cone picture corresponds exactly to the bonds of bonds of ... picture.

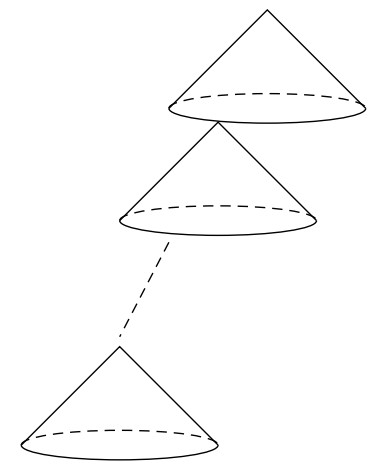

Figure 4: Several levels of cone structure

This motivates the definition of an extended higher category which we call a hypercategory, basically considered as sheaves on stratified sets:

$$
\operatorname{Sh}(\text { Strat }) \text {. }
$$

The details will be developed with D. Ayala in future work.

This is an interesting implementation of the more general hyperstructure architectures. Cone structures lead to decompositions and stratifications which again lead to hyperstructures and bonds, see Baas (2013b). Their representations lead to extended field theories.

\section{Compositions of BOnds}

In the study of collections of objects we emphasize the general notion of bonds including relations, functions and morphisms. We get richer structures when we have composition rules of various types of bonds. Such compositions should take into account the higher order architecture giving bonds a level structure.

We experience this situation in higher categories where we want to compose morphisms of any order. Suppose that we are given two $n$ morphisms $f$ and $g$. They may not be compatible at level $n$ for composition in the sense that

$$
\operatorname{target}(f)=\operatorname{source}(g) \text {. }
$$


But in a precise way we can iterate source and target maps to get down to lower levels, and it may then happen that at level $p$ we have

$$
\operatorname{target}_{p}^{n}(f)=\operatorname{source}_{p}^{n}(g) \text {. }
$$

Hence composition makes sense at level $p$ and we write the composition rule as

and the composed object as

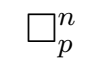

$$
f \square_{p}^{n} g .
$$

In a similar way we can introduce composition rules for bonds in a general hyperstructure $\mathscr{H}$. Let $a_{n}$ and $b_{n}$ be bonds at level $n$ in $\mathscr{H}$. Then we get to the lower levels via the boundary maps

$$
\partial_{i}: X_{i+1} \rightarrow \mathscr{P}\left(X_{i}\right)
$$

and search for compatibility in the sense that

$$
\partial_{p} \circ \cdots \circ \partial_{n-1}\left(a_{n}\right)=\partial_{p} \circ \cdots \circ \partial_{n-1}\left(b_{n}\right)
$$

or we may just require a weaker condition like

$$
\partial_{p} \circ \cdots \circ \partial_{n-1}\left(a_{n}\right) \cap \partial_{p} \circ \cdots \circ \partial_{n-1}\left(b_{n}\right) \neq \emptyset
$$

in order to have a composition defined:

$$
a_{n} \square_{p}^{n} b_{n}
$$

For bonds in a hyperstructure we may even compose bonds at different levels: $a_{m}, b_{n}$ compatible at level $p$ via boundary maps, allow us to define

$$
a_{m}{ }^{m} \square_{p}^{n} b_{n}
$$

as an $m$-bond for $m \geq n$. Compositional rules are needed and will appear elsewhere.

Composition may be thought of as a kind of geometric gluing. We consider the bonds as spaces, binding collections of families of subspaces, these again being bonds, etc. By the "boundary" maps we go down to a level where these are compatible, gluable bond spaces along which we may glue the bonds within the type of spaces we consider.

Therefore hyperstructures offer the framework for a new kind of higher order gluing in which the level architecture plays a major role. We will pursue this in the next section.

\section{Higher order GLUing AND EXTENDing SHEAVES}

Hyperstructures are useful tools in passing from local situations to global ones in collection of objects. In this process the level structure is important. We will here elaborate the discussion of multilevel state systems in Baas (2013b).

In mathematics we often consider situations locally at open sets covering a space and then glue together basically in one stroke - meaning 
there are just two levels local and global, no intermediate levels. In many situations dominated by a hyperstructure this is not sufficient. We need a more general hyperstructured way of passing from local to global in general collections.

Let us offer two of our intuitions regarding this process. Geometrically we think of a multilevel nested family of spaces, like manifolds with singularities represented by manifolds with multinested boundaries or just like higher dimensional cubes with iterated boundary structure (corners, edges,...). With two such structures we may then glue at the various levels of the nesting (Figure 5).

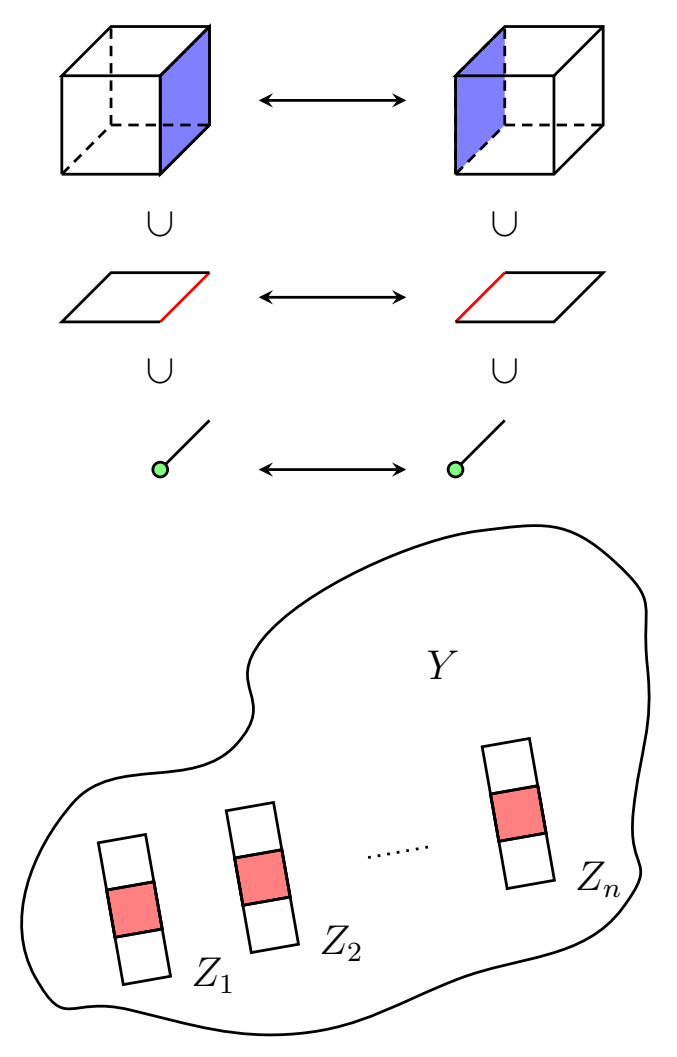

FigURE 5: Gluing possibility at various levels

Furthermore, study how states and properties may be "globalized", meaning putting local states coherently together to global states.

Biological systems are put together by multilevel structures from cells into tissues, organs etc. constituting an organism. Much of biology is about understanding how cell-states determine organismic states. The hyperstructure concept is in fact inspired by biological systems.

In order to extend the discussion of multilevel state systems in Baas (2013b) we need to generalize and formulate in a hyperstructure context the following mathematical notions (see for example Mac Lane and Moerdijk (1994)): 
- Sieve

- Grothendieck Topology

- Site

- Presheaf

- Sheaf

- Descent

- Stack

- Sheaf cohomology

Let us start with a given hyperstructure

$$
\begin{aligned}
\mathscr{H}:: & \left\{X_{0}, \ldots, X_{n+1}\right\} \\
& \left\{\Omega_{0}, \ldots, \Omega_{n}\right\} \\
& \left\{B_{0}, \ldots, B_{n}\right\} \\
& \left\{\partial_{0}, \ldots, \partial_{n}\right\}
\end{aligned}
$$

We will now suggest a series of new definitions.

Definition 1: A sieve on $\mathscr{H}$ is given as follows: At the lowest level $X_{0}$ a sieve $\mathcal{S}$ on a bond $b_{0}\left(=b_{0}\left(S_{0}, \omega_{0}\right)\right)$ is given by families of bonds $\left\{b_{o}^{j_{0}}\right\}$ (covering families) and $b_{1}$ 's such that

$$
b_{1}\left(\left\{b_{o}^{j_{0}}\right\}, b_{0}\right),
$$

$b_{0}$ may also be replaced by a family of bonds.

Bond composition with $\left\{b_{0}^{j_{0}}\right\}$ will produce new families in the sieve.

A sieve on $\mathscr{H}$ is then a family of such sieves $\left(\mathcal{S}_{k}\right)_{k=1, \ldots, n}$ - one for each level.

We postpone connecting the levels until the definition of a Grothendieck topology, but this could also have been added to the sieve definition.

Definition 2: A Grothendieck topology on $\mathscr{H}$ is given as follows: First we define a Grothendieck topology for each level of bonds. Consider level 0: to every bond $b_{0}$ we assign a collection of sieves $J\left(b_{0}\right)$ such that

i) (maximality), the maximal sieve on $b_{0}$ is in $J\left(b_{0}\right)$

ii) (stability), let $S \in J\left(b_{0}\right), b_{1}\left(b_{0}^{\prime}, b_{0}\right)$, then in obvious notation

$$
b_{1}^{*}(S) \in J\left(b_{0}^{\prime}\right)
$$

iii) (transitivity), let $S \in J\left(b_{0}\right)$ and $R$ any sieve on $b_{0}, b_{0}^{\prime}$ an element of a covering family in $S, b_{1}^{*}(R) \in J\left(b_{0}^{\prime}\right)$ for all $b_{1}$ with $b_{1}\left(b_{0}^{\prime}, b_{0}\right)$, then $R \in J\left(b_{0}\right)$.

We call $J\left(b_{0}\right)$ is a $J$-covering of $b_{0}$.

This gives a Grothendieck topology for all levels of bonds, and we connect them to a structure on all of $\mathscr{H}$ by defining in addition an assignment $J$ of $\left(b_{0}, \ldots, b_{n}\right)$ where $b_{i} \in \partial_{i} b_{i+1}$. 
$J\left(b_{0}, \ldots, b_{n}\right)$ consists of families of sieves $\left\{b_{o}^{j_{0}}\right\} \in J\left(b_{0}\right), \ldots,\left\{b_{n}^{j_{n}}\right\} \in$ $J\left(b_{n}\right)$ and bonds

$$
\beta_{1}, \ldots, \beta_{n+1}
$$

such that

$$
\beta_{1}\left(b_{0},\left\{b_{o}^{j_{0}}\right\}\right), \ldots, \beta_{n+1}\left(b_{n},\left\{b_{n}^{j_{n}}\right\}\right)
$$

and $b_{i}^{j_{i}} \in \partial_{i} b_{i+1}^{j_{i+1}}$. In a diagram we have

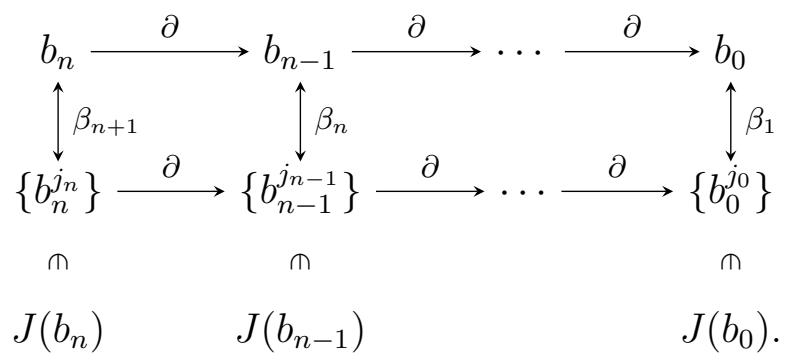

Clearly there are many possible choices of Grothendieck topologies, and they will be useful in the gluing process and the creation of global states. Examples will be discussed elsewhere, our main point here is to outline the general ideas.

Definition 3: $(\mathscr{H}, J)$ is called a hyperstructure site when $J$ is a Grothendieck topology on the hyperstructure $\mathscr{H}$.

The categorical notion of a presheaf we modify as follows. We think of the $\Omega_{i}$ 's in $\mathscr{H}$ as state (property, field, dataset, etc.) assignments in a structure preserving way, covering cellular type (pre)-sheaves as well. These assignments would be different from the ones used in the construction of $\mathscr{H}$. As in Baas (2013b) we may let them take values in hyperstructures of states

$$
\mathscr{S}=\left\{\mathscr{S}_{0}, \mathscr{S}_{1}, \ldots, \mathscr{S}_{n}\right\}
$$

$\mathscr{S}_{i}$ being a hyperstructure such that

$$
\begin{aligned}
& \Omega_{0} \text { takes values in } \mathscr{S}_{n} \\
& \vdots \\
& \Omega_{i} \text { takes values in } \mathscr{S}_{n-i} \\
& \vdots \\
& \Omega_{n} \text { takes values in } \mathscr{S}_{0}
\end{aligned}
$$

and we assume that we have bond compatibility of the $\Omega_{i}$ 's and level connecting assignments $\delta_{i}$ ("dual" to the $\partial_{i}$ 's and acting on collections of bond "states") depending on the Grothendieck topology $J$ :

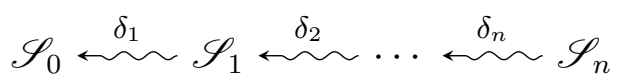


The $\delta_{i}$ 's may be functional assignments or relational, and the $\mathscr{S}_{i}$ 's often have an algebraic structure. In the simplest case all the $\mathscr{S}_{i}$ 's could just be Sets.

It should be pointed out that the $\Omega_{i}$ 's are already part of the $\mathscr{H}$ structure which we now combine with the Grothendieck topology. This will be useful in constructing new $\Omega$-states of global bonds. We consider the $\Omega_{i}$ 's as a kind of "level presheaves" and the $\delta_{i}$ 's giving a kind of "global matching families" — between levels in addition to levelwise matching. However, if we have "functional" assignment connectors $\hat{\delta}_{i}$ 's on $\mathscr{H}$ :

$$
\mathscr{S}_{0} \stackrel{\hat{\delta}_{1}}{\longleftarrow} \mathscr{S}_{1} \stackrel{\hat{\delta}_{2}}{\longleftarrow} \cdots \stackrel{\hat{\delta}_{n}}{\longleftarrow} \mathscr{S}_{n}
$$

means that we get a unique state of global bond objects (like an amalgamation for presheaves but here across levels in addition to levelwise amalgamation). Global bonds are "covered" as follows (see Baas $(2013 b))$

$$
\left\{b\left(i_{n}\right)\right\} \stackrel{\partial_{n-1}}{\longrightarrow}\left\{b\left(i_{n-1}, i_{n}\right)\right\} \stackrel{\partial_{n-2}}{\longrightarrow} \cdots \stackrel{\partial_{0}}{\longrightarrow}\left\{b\left(i_{0}, \ldots, i_{n}\right)\right\}
$$

and states are being levelwise globalized by

$$
\Omega_{n}\left(\left\{b\left(i_{n}\right)\right\}\right) \stackrel{\hat{\delta}_{1}}{\longleftarrow} \Omega_{n-1}\left(\left\{b\left(i_{n-1}, i_{n}\right)\right\}\right) \stackrel{\hat{\delta}_{2}}{\longleftarrow} \ldots \stackrel{\hat{\delta}_{n}}{\longleftarrow} \Omega_{0}\left(\left\{b\left(i_{0}, \ldots, i_{n}\right\}\right) .\right.
$$

With a slight abuse of notation we write this as

$$
\Omega:(\mathscr{H}, J) \rightarrow \mathscr{S}
$$

and define $\Omega=\left\{\Omega_{i}\right\}$ as a "presheaf" on $(\mathscr{H}, J)(\operatorname{Pre}(\mathscr{H}, J))$ and when

$$
\Delta=\left\{\hat{\delta}_{i}\right\}
$$

exists we have a unique global bond state. This is like a sheafification condition and we call $(\Delta, \Omega)$ a globalizer of the site $(\mathscr{H}, J)$ with respect to $\Omega$.

$\Omega$ with $\Delta$ extends the sheaf notion here, gluing within levels and between levels.

A globalizer is a kind of higher order or hyperstructured sheaf covering all the levels.

The existence of $\Delta$ contains the global gluing data and hence corresponds to what is often called descent conditions and the hyperstructure collection $\mathscr{S}$ extends the notion of a stack over $\mathscr{H}$.

Since $\Omega$ is already built into the structure of $\mathscr{H}$ we may consider $\Omega$ and $(\Omega, \Delta)$ as "internal" presheaves and sheaves. However, we may alternatively define an "external" assignment

$$
\Lambda:(\mathscr{H}, J) \rightarrow \mathscr{S}
$$


where $\Lambda=\left\{\Lambda_{i}\right\}$ and

$$
\begin{aligned}
& \Lambda_{0} \text { takes values in } \mathscr{S}_{n} \\
& \begin{array}{c}
\vdots \\
\Lambda_{i} \text { takes values in } \mathscr{S}_{n-1}
\end{array}
\end{aligned}
$$

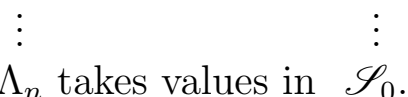

$\Lambda$ is being globalized in the same way as $\Omega$, the "boundary decomposition"

$$
\left\{b\left(i_{n}\right)\right\} \stackrel{\partial_{n-1}}{\longrightarrow}\left\{b\left(i_{n-1}, i_{n}\right)\right\} \stackrel{\partial_{n-2}}{\longrightarrow} \cdots \stackrel{\partial_{0}}{\longrightarrow}\left\{b\left(i_{0}, \ldots, i_{n}\right)\right\}
$$

gives rise to

$$
\Lambda_{n}\left(\left\{b\left(i_{n}\right)\right\}\right) \stackrel{\delta_{1}^{\prime}}{\longleftarrow} \Lambda_{n-1}\left(\left\{b\left(i_{n-1}, i_{n}\right)\right\}\right) \stackrel{\delta_{2}^{\prime}}{\longleftarrow} \cdots \stackrel{\delta_{n}^{\prime}}{\longleftarrow} \Lambda_{0}\left(\left\{b\left(i_{0}, \ldots, i_{n}\right\}\right) .\right.
$$

When

$$
\Lambda:(\mathscr{H}, J) \rightarrow \mathscr{S}
$$

is an "external" presheaf and $\Delta=\left\{\delta_{i}^{\prime}\right\}$ exists we define $(\Delta, \Lambda)$ to be an "external" globalizer.

To a site $(\mathscr{H}, J)$ we may also form a hyperstructure in a suitable way

$$
\operatorname{Glob}(\mathscr{H}, J)
$$

of globalizers (internal or external) corresponding to a Grothendieck topos. If $\mathscr{H}$ is just a one level categorical structure and $\mathscr{S}=$ Sets, then

$$
\operatorname{Glob}(\mathscr{H}, J)=\operatorname{Sh}(\mathscr{H}, J) .
$$

being an $n$-category of some kind is another special case.

This framework is very useful in formulating and studying local to global situations, and extends the discussion started in Baas (2013b), where we as examples studied globalizers given by compositions of mappings.

In view of our discussion in section 5 this leads to the following chain of structures

$$
\operatorname{Sh}(M f d) \longleftrightarrow \operatorname{Sh}(\text { Strat }) \longleftrightarrow \operatorname{Glob}(\mathscr{H} \text {-site }) .
$$

The concepts introduced here also paves the way for new types of cohomology of the basic collection or set, say $Z$, with "coefficients" in "presheaves" and "globalizers":

$$
H^{*}(Z ; \mathscr{P}) \text { and } H^{*}(Z ; \mathscr{G})
$$

$\mathscr{P} \in \operatorname{Pre}(\mathscr{H}, J), \mathscr{G} \in \operatorname{Glob}(\mathscr{H}, J)$ - all to be defined in a suitable setting with $\mathscr{S}$ having an algebraic structure, to be discussed elsewhere. 
In a hyperstructure the "boundary" maps $\partial_{i}$ connect the levels. A hyperstructure on a set or collection $Z, \mathscr{H}(Z)$, may be thought of as a structural kind of resolution or "chain complex" by adding suitable signs to the boundary operator. $\mathscr{H}(Z)$ may be thought of as a structure on $Z$ or a structure measure. These structures may lead to new cohomological notions beyond $\mathscr{P}$ and $\mathscr{G}$.

If the $\mathscr{S}_{i}$ 's have a tensor product it is natural to require:

$$
\hat{\delta}_{n-i+1}: \otimes\left\{\Omega_{i-1}\left(\left\{b_{i-1}\right\}\right)\right\} \longrightarrow \Omega_{i}\left(b_{i}\right)
$$

relating our globalizers to factorization algebras and quantum field theories (Baas (2013b)). The breakdown of a globalizer may lead to disorganized states. In cells in an organism this may be related to cancerous states, see also Baas (2006). Globalizers represent an organizational way to get global properties and states of general collections of objects.

In hyperstructures the release of properties, energy or creation of states at one level may facilitate new releases or creations at the higher levels. This will continue until often an optimum is reached at the top (global) level. This is an analogy with the gluing of states in Globs. The mathematical details of this construction will be followed up elsewhere. Our main purpose here is to show how hyperstructures may be used in obtaining global states, properties, fields or objects from local ones.

\section{BONDS AND BRIDGES}

Often in mathematics and science one would like to transfer a structure in one context to a similar structure in another context. Hyperstructures are useful tools in such situations transfering structures via higher bonds. This has been discussed in Baas (2013b), but we will elaborate on the theme.

Putting a hyperstructure on a collection may be useful in the study and use of the collection. Sometimes one may transfer the structure to another situation and use it there in order to get new results otherwise difficult to detect or prove.

If we have a collection $X$ and a hyperstructure on it, $\mathscr{H}(X)$, and $X$ is "related" in some way to another collection $Z$, we may let the relations propagate throught $\mathscr{H}$ in order to form a new hyperstructure $\mathscr{H}(Z)$ as discussed in Baas (2013b), see Figure6. 


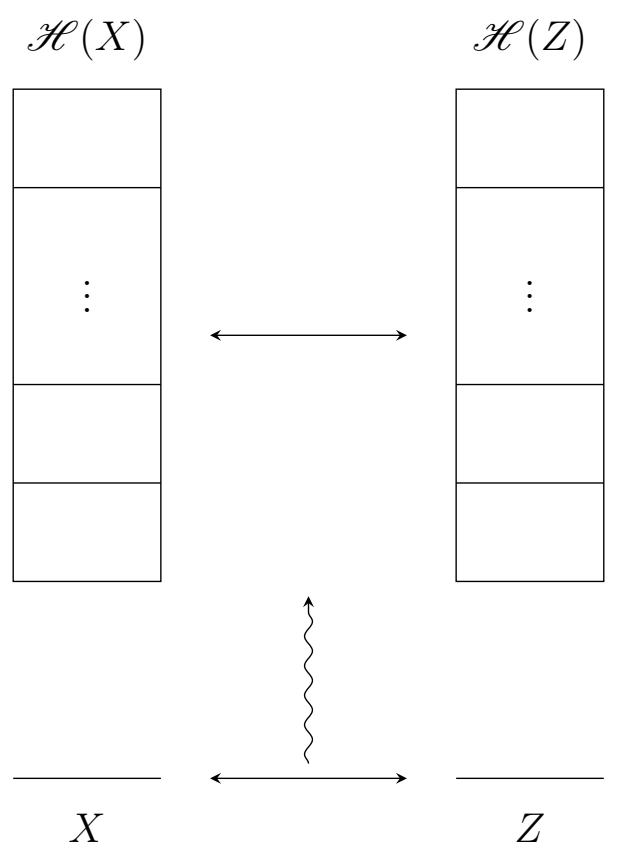

FiguRE 6: Structural transfer

This induced transfer of structure is essentially a new kind of bond in a hyperstructure embracing the two $(\mathscr{H}(X)$ and $\mathscr{H}(Z))$ as indicated in Figure 7.

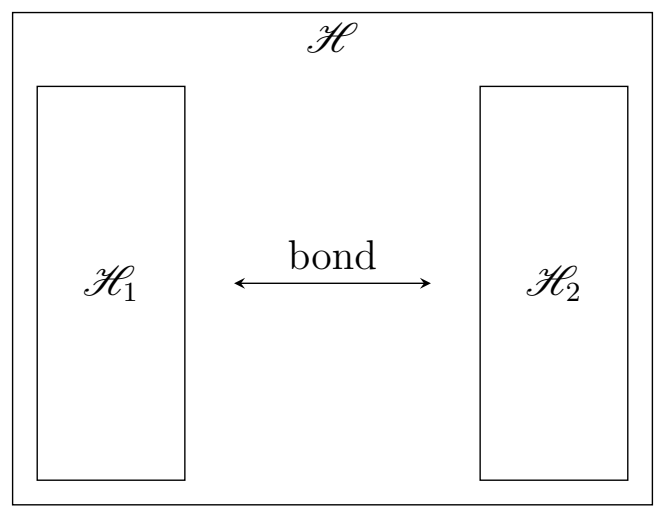

FiguRE 7: A hyperstructure bridge 


\begin{tabular}{|c|}
\hline $\mathscr{H}_{R_{0}}^{n}(Z)$ \\
\hline \\
$\vdots$ \\
\hline $\mathscr{H}_{R_{0}}^{1}(Z)$ \\
\hline $\mathscr{H}_{R_{0}}^{0}(Z)=Z$ \\
\hline
\end{tabular}

$\mathscr{H}_{R_{0}}(Z)$

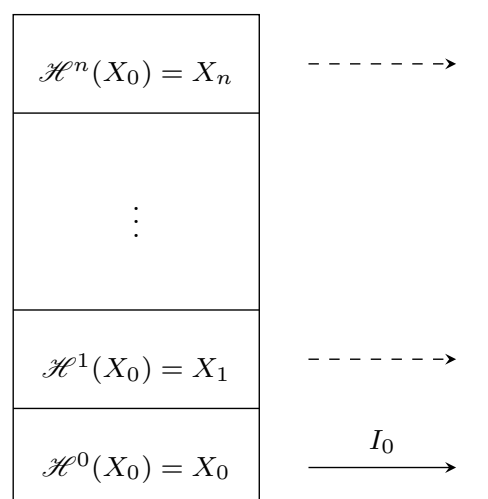

$\mathscr{H}\left(X_{0}\right)$

State hyperstructure:

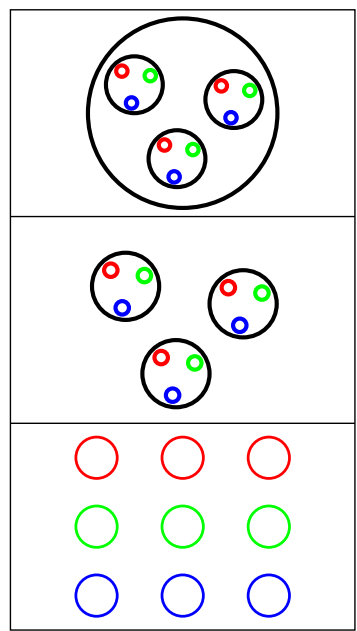

$Z=9$ particle states

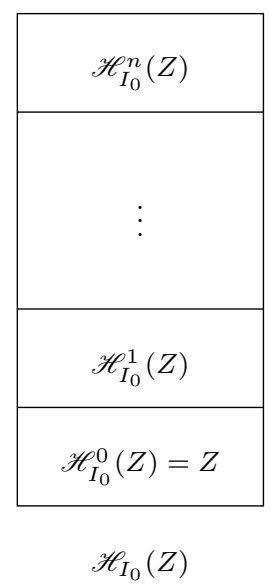

Link hyperstructure:

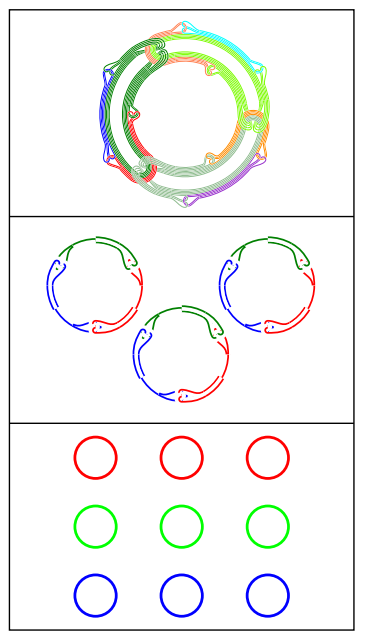

$X_{0}=9$ rings

FiguRE 8: Transfer of structures via hyperstructures

Such a bond will act as a bridge between the two structures transferring at the various levels.

An example of such transfer of structure is the following discussed in Baas (2013b):

A finite set of rings in three dimensional space may be given an $\mathscr{H}$ structure with a levelwise Brunnian property: the rings at any level are linked such that if one is removed, the rest become unlinked. Then we may consider a (quantum) particle system and by representing particles by rings we may give the particle system a similar $\mathscr{H}$-structure with the Brunnian property: if at any level a certain number of particle collections interact in such a way that if one collection is removed, the remaining collections do not interact. The representation 
ring $\longrightarrow$ particle

induces the bridge of hyperstructures, see Figure 8 .

Bridges have been discussed in many interesting ways by Caramello (2014). The main idea being transfer of information between two different sites via categories or toposes of sheaves as follows: given two sites

$$
\left(\mathscr{C}_{1}, J_{1}\right) \text { and }\left(\mathscr{C}_{2}, J_{2}\right)
$$

We may have the situation

$$
\left(\mathscr{C}_{1}, J_{1}\right) \longrightarrow \operatorname{Sh}\left(\mathscr{C}_{1}, J_{1}\right) \simeq \operatorname{Sh}\left(\mathscr{C}_{2}, J_{2}\right) \longrightarrow\left(\mathscr{C}_{2}, J_{2}\right)
$$

where $\simeq$ means (Morita-type) equivalence. If such an equivalence exists it represents a bridge for transfer of information between $\left(\mathscr{C}_{1}, J_{1}\right)$ and $\left(\mathscr{C}_{2}, J_{2}\right)$. In our terminology we think of the equivalence as a bond in the hyperstructure or the category of Grothendieck toposes.

In the hyperstructure setting this corresponds to given two $\mathscr{H}$-sites $\left(\mathscr{H}_{1}, J_{1}\right)$ and $\left(\mathscr{H}_{2}, J_{2}\right)$ with a bridge or bond of the globalizer hyperstructures:

$$
\left(\mathscr{H}_{1}, J_{1}\right) \longrightarrow \operatorname{Glob}\left(\mathscr{H}_{1}, J_{1}\right) \simeq \operatorname{Glob}\left(\mathscr{H}_{2}, J_{2}\right) \longrightarrow\left(\mathscr{H}_{2}, J_{2}\right)
$$

where $\simeq$ means a suitable equivalence or bond.

In general when

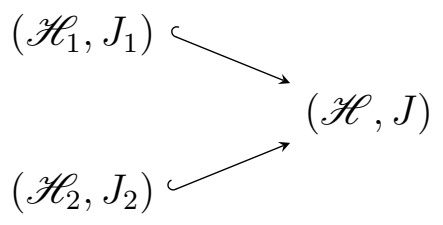

one may transfer structures via the top level in $\mathscr{H}$ and similarly for states via

$$
\operatorname{Glob}\left(\mathscr{H}_{1}, J_{1}\right) \longrightarrow \operatorname{Glob}(\mathscr{H}, J) .
$$

Another possible type of bridge using hyperstructures would be to introduce a "Brave New $\mathscr{H}$-algebra" where equalities (and hence equations) are being replaced by bonds in hyperstructures. This extends homotopical algebra (homotopy bonds) and gives many new perspectives to be explored.

\section{Organizing COLleCtions}

When working with collections of objects it is often desirable to make new collections from old ones. One may break up collections to new 
ones, break up objects to more objects (fission) or amalgamate objects and collections (fusion). As we have pointed out organizing collections is useful in detecting and creating properties and structure, controlling actions and making constructions, classifying and achieving goals. Our thesis is that hyperstructured architectures are very useful in doing this.

In this setting fusion and fission are dual. In a fusion process one takes the given collection as the lowest level, and in a fission process we let the collection represent the top level. This is analogous to preparing a collection for analysis or synthesis. Our collections could consist of any types of objects, be sets, spaces (topological and metric spaces, manifolds, simplicial spaces, varieties,...), groupoids, even hyperstructures themselves. To illustrate our ideas we will here basically consider fusion-type processes.

Putting a hyperstructure on a collection in such a way that the collection is represented as the lowest level of the hyperstructure is essentially a fusion process in the sense that objects are levelwise being bound. Then local states and properties may propagate through the hyperstructure by globalizers to the top level. This represents a fusion of existing objects into collections of collections etc. determinded by the bonds. Such a fusion process may be induced by a hyperstructure on the collection or a hyperstructure on the ambient space of the collection which again will induce a hyperstructure on the collection. This may be illustrated as in Figure 9 .
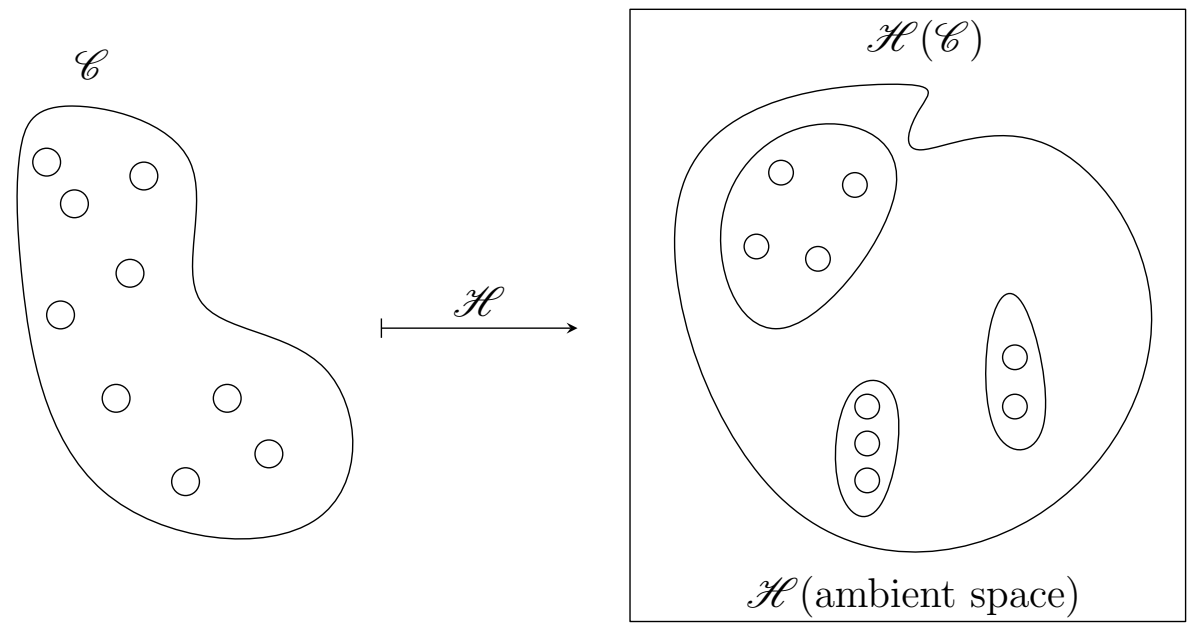

FiguRE 9: Fusion of a collection

The next basic step is the fusion of a collection of collections into a collection (Figure 10. Let us just consider two collections $\mathscr{C}_{1}$ and $\mathscr{C}_{2}$, the process defines a "fusion product":

$$
\left(\mathscr{C}_{1}, \mathscr{C}_{2}\right) \longmapsto \mathscr{C}_{1} \square_{\mathscr{H}} \mathscr{C}_{2}
$$



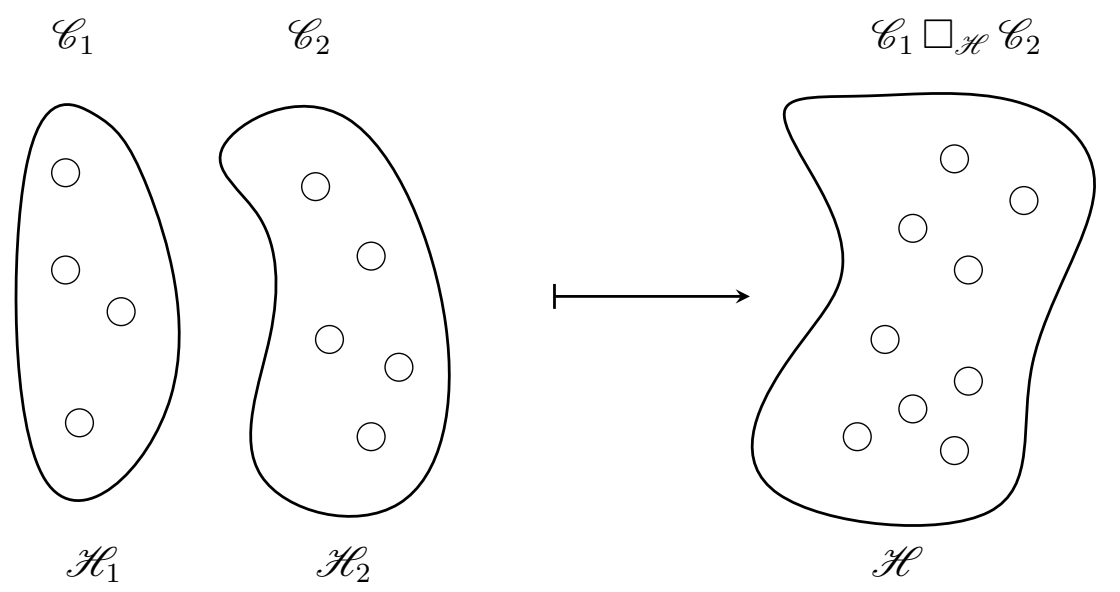

FiguRE 10: Fusing collections

The new thing to study is that $\square_{\mathscr{H}}$ depends on a hyperstructure ( $\mathscr{H}$-structure) on $\mathscr{C}_{1}$ and $\mathscr{C}_{2}$ in order to obtain the fusion. They may possibly be different and $\square_{\mathscr{H}}$ stands as a generic notion. States and properties may then be built in such that $\mathscr{C}_{1} \square \mathscr{H}_{2} \mathscr{C}_{2}$ is a collection with a derived global state or property

$$
\omega\left(\mathscr{C}_{1} \square \mathscr{H}_{2} \mathscr{C}_{2}\right)
$$

determined from $\omega\left(\mathscr{C}_{1}\right)$ and $\omega\left(\mathscr{C}_{2}\right)$ via globalizers in $\mathscr{H}$. Such a process may reflect a "release" of properties of the bonds - like for example a change from high to low energy levels.

The second type of fusion to be considered is when objects fuse into new types of objects, hence we form a new collection $\mathscr{C}$ from $\mathscr{C}^{\prime}$ :
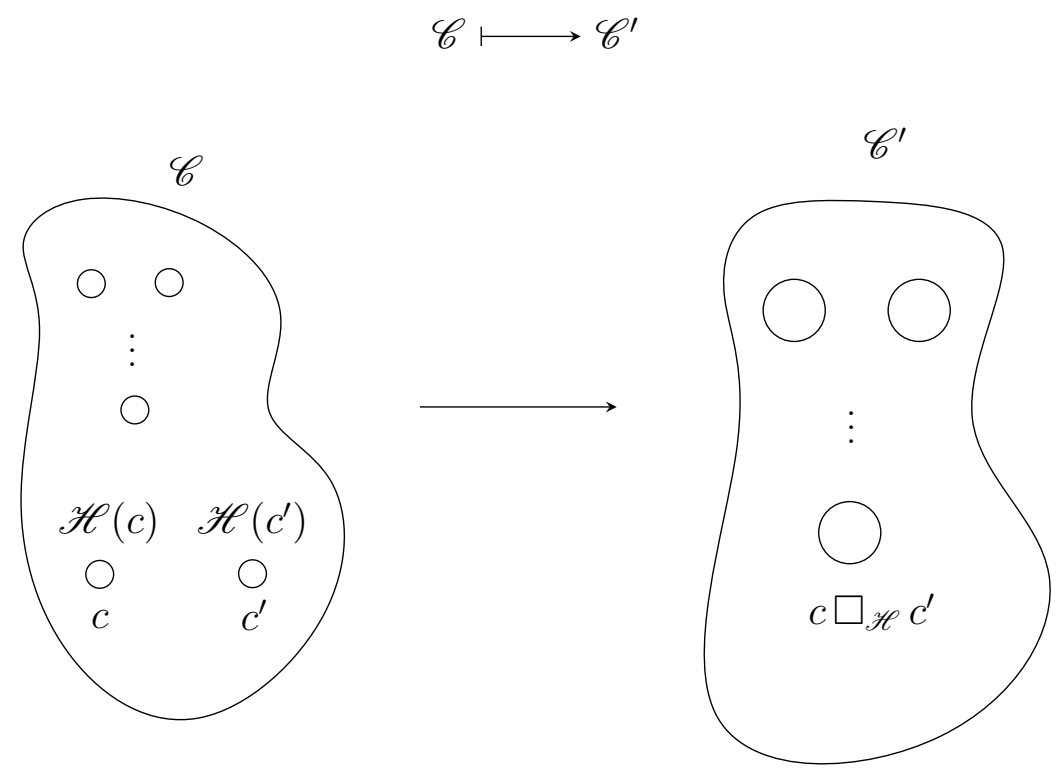

Figure 11: Object fusion in a collection by object hyperstructures 
When such a process is desired one may assign a hyperstructure $\mathscr{H}(c)$ to each object in the collection, $c \in \mathscr{C}$ (Figure 11). $\mathscr{H}(c)$ may be induced from a hyperstructure $\mathscr{H}_{A}$ of an ambient space $A$. There may also be an $\mathscr{H}(\mathscr{C})$ on the total collection and they may possibly all interact in order to create globalizers with desired properties (for example like release of energy as in nuclear fusion). This applies not only to fusion of pairs, but to arbitrary collections (finite or infinite).

Fusion processes of this type give rise to many interesting aspects like introducing "energy" thresholds for fusion at each level and studying how the fusion process propagates through the $\mathscr{H}$-organized collection.

Our main point of view is that a hyperstructure on objects, collections or ambient space may facilitate fusion (and similar) processes, being an application of (see Baas (2013b)):

The Hyperstructure Principle: In order to study or use a collection of objects it is useful to put on a suitable Hyperstructure to reach a goal. Hyperstructures are tools for thought and tools for organizing complex information.

\section{TyPES, BONDS AND HYPERSTRUCTURES}

Type theory is a formal logical system intended to provide a framework for mathematical thinking and constructions. It has also turned out to be useful as a framework for high-level programming languages. In traditional type theory one may think of types as sets, but in a new approach V. Voevodsky and co-workers have introduced Homotopy Type Theory where one basically thinks of types as spaces and homotopy types, Voevodsky \& co (HTT 2013). Furthermore, to each type one may associate an $\infty$-groupoid. Voevodsky advocates building mathematics on $(\infty-)$ groupoids instead of sets.

As pointed out in (Baas 1996, Baas 2013a, Baas 2013b) we think of hyperstructures as in many ways reflecting evolutionary processes and the way we think and make things. Shortly they represent: Tools for Thought. This becomes quite apparant in higher order thinking when we have to consider several levels at a time. It may be that the human brain is basically designed to consider three levels at a time: where we are, the level above and the level below, see for example Koestler (1979). Beyond that we need a framework in which to reason, since comprehending higher order structures and hierarchies is vital in human thinking. We clearly see this in axiomatic systems (ZFC) for set theory.

In a lecture at IAS in Princeton March 26, 2014, V. Voevodsky suggested that a system adequate both for human reasoning and computer verifications should contain:

i) a formal deduction system

ii) providing meaning comprehensible to humans 
iii) a way for humans to encode mathematical ideas (like hierarchies).

We suggest that hyperstructures do this and may be extended to a new type theory. It is desirable that deductions are directly built into the system. This is the case for hyperstructures as we will show.

The simple idea is: Think of bonds as types. We have higher bonds (bonds of bonds ...) giving higher types. So higher order structures and hierarchies are built in. The semantics (meaning aspect) is built in via the $\Omega$ assignments ("states or properties").

The deducational part goes as follows: a bond $b_{1}$ at one level binds bonds at a lower level: $\left\{a_{o}^{i}\right\},\left\{b_{0}^{j}\right\}$.

Assume that for every bond $b_{1}$ and a given collection $\left\{a_{o}^{i}\right\}$ one can decide whether

$$
b_{1}\left(\left\{a_{o}^{i}\right\},\left\{b_{o}^{j}\right\}\right)
$$

holds for the collection $\left\{b_{o}^{j}\right\}$. If so, we say that $\left\{b_{o}^{j}\right\}$ can be deduced from $\left\{a_{o}^{i}\right\}$.

One may even allow an intermediate "proof" - family $\left\{c_{0}^{k}\right\}$, such that

$$
b_{1}\left(\left\{a_{0}^{i}\right\},\left\{c_{0}^{k}\right\},\left\{b_{0}^{j}\right\}\right) .
$$

Geometrically this may be thought of as a filling-in condition a la Kanconditions in homotopy theory. Oriented bonds may also be considered.

If the bonds are represented as spaces one may intuitively think of a proof as filling in the missing pieces of a decomposition (for example in a triangulation of a space). This will allow for a geometric way of thinking of deductions.

Universes of bonds of order $n$ will be bonds of order $(n+1)$. Since each bond governs a sub-hyperstructure one will have the association of a type $=$ bond $=A$, to a hyperstructure $\mathscr{H}_{A}$, similar to the $\infty$-groupoid association in homotopy type theory.

In the deduction both syntax and semantics play a role. Comprehension of (global) meaning comes from the existence of globalizers (global type sheaves). This is compatible with our point of view of hyperstructures as also describing evolutionary structures. For example in a biological system - an organism - a globalizer would assign a global biological state - useful in taking actions.

In hyperstructure types one may have a variety of "identity types",

$$
b_{1}\left(b_{0}^{i_{1}}\right), \quad b_{1}\left(b_{0}^{i_{1}}, b_{0}^{i_{2}}\right), \quad \ldots \quad, \quad b_{1}\left(b_{0}^{i_{1}}, \ldots, b_{0}^{i_{n}}\right)
$$

— if needed, motivated by geometric cobordisms.

Hyperstructures as introduced here are based on sets or collections. They exist in the universe of sets with structure. However, in the universe of homotopy type theory one may study $\infty$-groupoids with hyperstructures added on as additional structure. Or if a type theory of hyperstructures is taken as a basis for our thinking we would 
consider hyperstructures with other imposed hyperstructures. In general, whichever basic building blocks we choose for our formal thinking system, we should also in addition consider them equipped with hyperstructures.

In order to describe, encode and manipulate (mathematical) ideas in a formal system symbols are needed.

Meaningful symbol manipulations result in collections of symbols organized into a hyperstructure which may improve the encoding of many mathematical ideas. This allows for a symbol representation by higher dimensional shapes, not just one dimensional shapes and one dimensional concatenation. Symbols may be higher dimensional shapes and concatenation being replaced by higher order gluing in the form of bonds of symbols being bonds of bonds ... Symbol manipulation is then controlled by higher order bonds and rules for bond composition. We will return to these issues in future papers.

\section{Conclusion}

Using hyperstructures gives rise to a variety of higher order architectures on general collections of objects. We have argued that putting a hyperstructure on a collection or situation may be very useful, and new concepts have been introduced. Higher order gluing, bridges, fusion of collections and local to global situations have been discussed.

We think that the higher order architectures based on hyperstructures may in the future turn out to be very useful in many areas of science: mathematics, physics, chemistry, biology and neuroscience, computer science, economics and social science, engineering, systems science and architecture itself, as a matter of fact in all areas of human thought.

\section{ACKNOWLEDGEMENTS}

I would like to thank Marius Thaule for valuable technical assistance with the manuscript, and Andrew Stacey for the graphical production of Figure 2 and parts of Figure 8, The rest of the figures were made by Marius Thaule. Furthermore, I would also like to thank The Institute for Advanced Study, Princeton, NJ, USA for their kind hospitality during my stay there in 2013 when parts of this work were done.

\section{REFERENCES}

Baas, N.A. (1973). On bordism theory of manifolds with singularities. Mathematica Scandinavica, 33, 279-302.

Baas, N.A. (1994a). Emergence, hierarchies, and hyperstructures. In: C.G. Langton, ed. Artificial Life III, Santa Fe Institute Studies in the Sciences of Complexity. Langton: Addison-Wesley, 515-537.

Baas, N.A. (1994b). Hyperstructures as tools in nanotechnology. Nanobiology, 3(1), 49-60. 
Baas, N.A. (1996). A framework for higher order cognition and consciousness. In: S. Hameroff, A. Kaszniak and A. Scott, eds. Toward a science of consciousness. 633-648, The MIT Press, Cambridge.

Baas, N.A. (2006). Hyperstructures as abstract matter. Advances in Complex Systems, 9(3), 157-182.

Baas, N.A. (2009a). Hyperstructures, Topology and Datasets. Axiomathes, 19, 281295.

Baas, N.A. (2009b). New structures in complex systems. European Physical J. Special Topics, 178, 25-44.

Baas, N.A. (2013a). New states of matter suggested by new topological structures. Intern. J. of General Systems, 42(2), 137-169.

Baas, N.A. (2013b). On Structure and Organization: An Organizing Principle. Intern. J. of General Systems, 42(2), 170-196.

Baas, N.A, Fedorov, D.V., Jensen, A.S., Riisager, K., Volosniev, A.G., and Zinner, N.T. (2014) Higher-Order Brunnian Structures and Possible Physical Realizations, Physics of Atomic Nuclei, 77(3), 336-343.

Baas, N.A., and Seeman, N.C. (2012). On the chemical synthesis of new topological structures. J. of Mathematical Chemistry, 50, 220-232.

Bourbaki, N. Théorie des ensembles, Ch. 4, Structures, Hermann, Paris, 1966.

Caramello, O. (2014). http://www.oliviacaramello.com/Unification/Unification.htm [Accessed 16 June 2014].

Koestler, A. (1979). Janus: A Summing Up. Hutchinson, London.

Mac Lane, S., and Moerdijk, I. (1994). Sheaves in Geometry and Logic. SpringerVerlag, Heidelberg.

The Univalent Foundations Program (2013). Homotopy Type Theory: Univalent Foundations of Mathematics. http://homotopytypetheory.org/book [Accessed 16 June 2014]. 